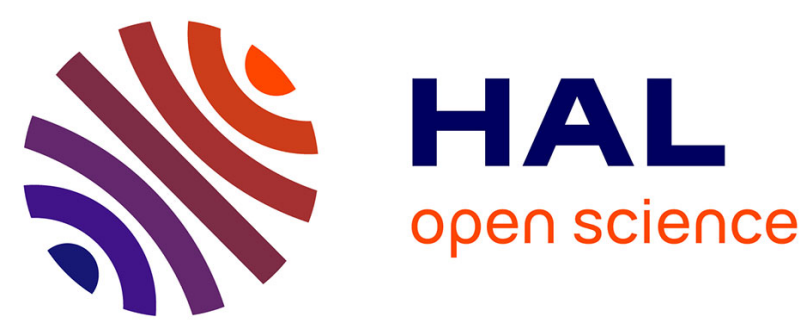

\title{
Performance Measure of Vehicle Onboard Vision System: An Interval Observer-based Approach
}

Sara Ifqir, Naïma Ait-Oufroukh, Dalil Ichalal, Saïd Mammar

\section{To cite this version:}

Sara Ifqir, Naïma Ait-Oufroukh, Dalil Ichalal, Saïd Mammar. Performance Measure of Vehicle Onboard Vision System: An Interval Observer-based Approach. 2018 IEEE International Conference on Vehicular Electronics and Safety (ICVES 2018), Sep 2018, Madrid, Spain. hal-01871100

\section{HAL Id: hal-01871100 https://hal.science/hal-01871100}

Submitted on 10 Sep 2018

HAL is a multi-disciplinary open access archive for the deposit and dissemination of scientific research documents, whether they are published or not. The documents may come from teaching and research institutions in France or abroad, or from public or private research centers.
L'archive ouverte pluridisciplinaire HAL, est destinée au dépôt et à la diffusion de documents scientifiques de niveau recherche, publiés ou non, émanant des établissements d'enseignement et de recherche français ou étrangers, des laboratoires publics ou privés. 


\title{
Performance Measure of Vehicle Onboard Vision System: An Interval Observer-based Approach
}

\author{
S. Ifqir*, N. Ait Oufroukh*, D. Ichalal* and S. Mammar* \\ * IBISC Laboratory, Univ Evry, Paris-Saclay University, Evry, France. \\ Email: \{sara.ifqir, naima.aitoufroukh, dalil.ichalal, said.mammar\}@univ-evry.fr
}

\begin{abstract}
Advanced Driver assistance systems that assist in safe vehicle guidance require a thorough understanding of the crossed environment. Because these systems rely on the vision sensor's ability to detect road boundaries and lane marking, these systems are extremely sensitive to road conditions. It is therefore important to take a critical look at their key aspects and evaluate their performance and accuracy. In this work, an evaluation framework to measure the performance of a vehicle onboard vision system using set-membership theory is presented. The proposed approach considers the changes that might occur in vehicle dynamics during the maneuver. The experiment design and evaluation of the proposed method is shown using field data acquired using an instrumented vehicle.
\end{abstract}

\section{INTRODUCTION}

In the recent years much research efforts in the intelligent transportation system community has been devoted to the topics of lane departure warning and lane keeping assistance [10]. Most of the developed technologies are based on a vision system mounted on the vehicle to detect road marking and boundaries. These vision-based systems suffer from performance limitations and work reliably only when lane markings are clearly visible, condition that is unlikely to be satisfied in adverse weather and lighting conditions. Therefore, it is necessary to evaluate the ability and performance of such systems.

In this paper, a framework to quantify the performance of the vehicle onboard vision systems is presented using setmembership estimation theory. The basic references for the technical development of the paper relies on interval estimation concept which have been recently investigated (see e.g., [8], [6], [11], [13], [4], [5]). First, an interval observer is used to robustly estimate the vehicle yaw rate and lateral velocity using a vision system measurement. The road curvature is treated as an unknown input and a linear adaptive tire model is considered to take into account the changes of the road adhesion. Afterwards, a second interval observer is built in order to generate guaranteed bounds on the road curvature. The evaluation methodology is then based on checking the consistency between the curvature measurement and the estimated interval. When an inconsistency is detected, then the measure is no longer reliable. The performance of the proposed algorithm is evaluated using vehicle field data, results show that the proposed scheme succeeds to appropriately estimate the upper and lower bounds of the road curvature and confirms the reliability of the vision system measurements. The remainder of the paper is structured as follows: Prelim- inaries for the synthesis of Interval Observer and for stability analysis using Multiple Quadratic Input-to-State Stable (ISS) Lyapunov Functions (MQLF) are given in section II. Section III introduces the lateral vehicle model with vision system measurement used for the interval observers design and simulation analysis. Section IV deals with the description of the proposed interval observer, its stability analysis using MQLF, and its design based on optimization problem involving Linear Matrix Inequalities (LMIs). The results of experimental validation based on real data acquired using an equipped prototype vehicle are presented in section V. Finally, Section VI concludes the paper.

\section{PRELIMINARIES}

Left and right endpoints of an interval $[x]$ will be denoted by $x^{-}$and $x^{+}$, respectively, such as $[x]=\left[x^{-}, x^{+}\right] . A>0$ (resp. $A<0$ ) denotes a matrix with positive (resp. negative) components and $A \succ 0$ (resp. $A \prec 0$ ) means that the matrix is positive (resp. negative) definite. $A^{T}$ and $A^{\dagger}$ denote respectively the transpose and the pseudo-inverse of the matrix $A$. The symbol $\mathcal{I}_{n}$ refers to identity matrix with dimension $n \times n$. Let a vector $x \in \mathbb{R}^{n}$ or a matrix $A \in \mathbb{R}^{n \times n}$, one denotes $\bar{x}=\max \{0, x\}, \underline{x}=\bar{x}-x$ or $\bar{A}=\max \{0, A\}$, $\underline{A}=\bar{A}-A$. In the rest of this paper, all inequalities must be understood element-wise.

Definition 1. [7] A real matrix $A$ is called Metzler matrix if all its elements outside the main diagonal are nonnegative.

Lemma 1. [8] A matrix $A$ is a Metzler if and only if there exist $\eta \in \mathbb{R}_{+}$such that $A+\eta \mathcal{I}_{n} \geq 0$.

Definition 2. [9] The following switched system:

$$
\dot{x}(t)=A_{\sigma(t)} x(t)+\delta_{\sigma(t)}(t)
$$

where $x \in \mathbb{R}^{n}$ is the state, $\sigma(t): \mathbb{R}^{+} \rightarrow \mathcal{I}=\{1,2, \ldots, N\}$ is the switching law, is said to be positive switched system if $A_{i}$ is a $n \times n$ Metzler matrix and $\delta_{i}(t) \geq 0, \forall i \in \mathcal{I}, \forall t \geq t_{0}$.

Definition 3. [8] An interval observer is a pair of estimators providing a lower and upper bounds $\left(x^{-}(t)\right.$ and $\left.x^{+}(t)\right)$ of the real state vector $x(t)$ such that $x^{-}(t) \leq x(t) \leq x^{+}(t)$, $\forall t \geq t_{0}$.

Lemma 3. [6] Let the vector $x \in \mathbb{R}^{n}$ be a variable vector with given bounds $x^{+}(t) x^{-}(t) \in \mathbb{R}^{n}$ such that $x^{-}(t) \leq x(t) \leq$ $x^{+}(t)$.

1) If $A \in \mathbb{R}^{n \times n}$ is a constant matrix, then

$$
\bar{A} x^{-}-\underline{A} x^{+} \leq A x \leq \bar{A} x^{+}-\underline{A} x^{-}
$$


2) If $A \in \mathbb{R}^{n \times n}$ is a variable such that $A^{-} \leq A \leq A^{+}$for some $A^{-}, A^{+} \in \mathbb{R}^{n \times n}$, then

$$
\begin{gathered}
\underline{A}^{+} \underline{x}^{+}-\bar{A}^{+} \underline{x}^{-}-\underline{A}^{-} \bar{x}^{+}+\bar{A}^{-} \bar{x}^{-} \leq A x \leq \\
\bar{A}^{+} \bar{x}^{+}-\underline{A}^{+} \bar{x}^{-}-\bar{A}^{-} \underline{x}^{+}+\underline{A}^{-} \underline{x}^{-}
\end{gathered}
$$

Definition 4. [5] For a switching signal $\sigma(t)$ and any $t_{2}>t_{1}>$ $t_{0}$, let $N_{\sigma}\left(t_{1}, t_{2}\right)$ be the number of switching over the interval $\left[t_{1}, t_{2}\right)$. If the condition $N_{\sigma}\left(t_{1}, t_{2}\right) \leq N_{0}+\frac{\left(t_{2}-t_{1}\right)}{\tau_{a}}$ holds for $N_{0} \geq 1, \tau_{a}>0$, then $N_{0}$ and $\tau_{a}$ are called the chatter bound and the average dwell time respectively.

Lemma 4. [11] Consider the switched system (1) and let $\varepsilon>0$. Suppose that there exist smooth functions $V_{\sigma(t)}: \mathbb{R}^{n} \rightarrow \mathbb{R}$, where $V_{\sigma(t)}(x(t))$ switches among $V_{i}(x(t))=x^{T}(t) Q_{i} x(t)$ in accordance with the piecewise constant switching law $\sigma(t)$. Then, if there exist $\gamma>0, \beta>\alpha>0$ such that for each $\sigma(t)=i$, the following conditions hold:

$$
\begin{aligned}
& \alpha\|x(t)\| \leq V_{i}(x(t)) \leq \beta\|x(t)\| \\
& \dot{V}_{i}(x(t))<-\varepsilon V_{i}(x(t))+\gamma\left\|\delta_{i}\right\|
\end{aligned}
$$

then the system (1) is Input-to-State Stable with respect of the additive term $\delta_{\sigma(t)}$ for any switching signal with Average Dwell Time

$$
\tau_{a} \geq \tau_{a}^{*}=\frac{\ln (\mu)}{\varepsilon}
$$

where $\mu=\frac{\beta}{\alpha}$.

Proof. Due to space limitation reasons, the proof is omitted. It can be found in [12].

\section{VEHICLE MODEL DESCRIPTION}

\section{A. Vehicle Lateral Dynamics}

In this work, a simple model known as the bicycle model (Fig. 1) is used for vision system evaluation process. This model describes the vehicle yaw and lateral motions [1], the state equations have the following form:

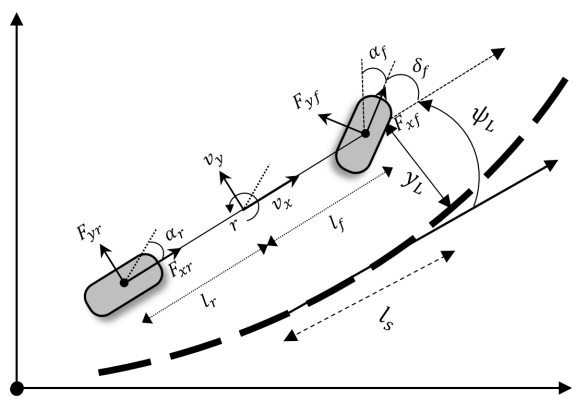

Fig. 1. Bicycle model and vision system measurement.

$$
\left\{\begin{array}{l}
m \dot{v}_{y}+m v_{x} r=F_{y f}+F_{y r} \\
I_{z} \dot{r}=l_{f} F_{y f}-l_{r} F_{y r}
\end{array}\right.
$$

where description of vehicle dynamics parameters is given in Table 1.

The lateral forces $F_{y f}$ and $F_{y r}$ are nonlinear and functions of the wheel sideslip angle ( [3], [2]). Using the so-called Pacejka magic formula [3], and under assumption of small
TABLE I

VeHICLE DYNAMICS AND VISION SYSTEM PARAMETERS

\begin{tabular}{ll}
\hline Lateral Dynamics & \\
\hline$F_{y r}, F_{y r}$ & Lateral tire force of front and rear tires \\
$v_{y}, v_{x}$ & Lateral and longitudinal velocities $\left(\mathrm{m}_{\mathrm{s}} \mathrm{s}^{-1}\right)$ \\
$r$ & Yaw rate $\left(\mathrm{rad} . \mathrm{s}^{-1}\right)$ \\
$\delta_{f}$ & Front steering angle $(\mathrm{rad})$ \\
$c_{f}, c_{r}$ & Cornering stiffness of front and rear tires $\left(\mathrm{N} . \mathrm{rad}^{-1}\right)$ \\
$l_{f}, l_{r}$ & Distances from front and rear axle to the CG $(\mathrm{m})$ \\
$m$ & Vehicle mass $(\mathrm{kg})$ \\
$I_{z}$ & Moment of inertia $\left(\mathrm{kg}^{2} \mathrm{~m}^{2}\right)$ \\
\hline Vision System & \\
\hline$y_{L}$ & Offset displacement at a look ahead distance $(\mathrm{m})$ \\
$\psi_{L}$ & Angular displacement at a look ahead distance $(\mathrm{rad})$ \\
$l_{s}$ & Look ahead distance $(\mathrm{m})$ \\
$\rho$ & Road curvature $\left(\mathrm{m}^{-1}\right)$ \\
$\beta$ & Sideslip angle $(\mathrm{rad})$
\end{tabular}

sideslip angle variation, lateral forces are taken to be linear and expressed as follows:

$$
F_{y f}=c_{f}\left(\delta_{f}-\frac{v_{y}}{v_{x}}-\frac{l_{f}}{v_{x}} r\right), \quad F_{y r}=c_{r}\left(-\frac{v_{y}}{v_{x}}+\frac{l_{r}}{v_{x}} r\right)
$$

In the proposed model, it is assumed that the available measurements are yaw rate $r$, longitudinal velocity $v_{x}$ and front steering angle $\delta_{f}$. Gathering equations (7) and (8) and chosen $v_{y}$ and $r$, as state variables, leads to the following state-space representation:

$$
\left[\begin{array}{c}
\dot{v}_{y} \\
\dot{r}
\end{array}\right]=\left[\begin{array}{cc}
-\frac{c_{f}+c_{r}}{m v_{x}} & \frac{c_{r} l_{r}-c_{f} l_{f}}{m v_{x}}-v_{x} \\
\frac{c_{r} l_{r}-c_{f} l_{f}}{I_{z} v_{x}}-\frac{c_{r} l_{r}^{2}+c_{f} l_{f}^{2}}{I_{z} v_{x}}
\end{array}\right]\left[\begin{array}{c}
v_{y} \\
r
\end{array}\right]+\left[\begin{array}{c}
\frac{c_{f}}{c_{f}^{m}} \\
\frac{c_{f} l_{f}}{I z}
\end{array}\right] \delta_{f}
$$

\section{B. Vision system dynamics}

The vision system model describes the evolution of the angular and lateral displacements of the vehicle from the centerline at a particular look-ahead distance $l_{s}$ (Fig. 1). The equations describing the vision system model are given by

$$
\left\{\begin{array}{l}
\dot{\psi}_{L}=r-v_{x} \rho \\
\dot{y}_{L}=v_{y}+v_{x} \psi_{L}+l_{s}\left(r-v_{x} \rho\right)
\end{array}\right.
$$

The system (10) can be rewritten in the following state representation form:

$$
\left[\begin{array}{c}
\dot{\psi}_{L} \\
\dot{y}_{L}
\end{array}\right]=\left[\begin{array}{cc}
0 & 0 \\
0 & v_{x}
\end{array}\right]\left[\begin{array}{l}
\psi_{L} \\
y_{L}
\end{array}\right]+\left[\begin{array}{cc}
0 & 1 \\
1 & l_{s}
\end{array}\right]\left[\begin{array}{c}
v_{y} \\
r
\end{array}\right]+\left[\begin{array}{c}
-v_{x} \\
-l_{s} v_{x}
\end{array}\right] \rho
$$

The vision systems parameters are described in Table 1 .

\section{Combined Model and Problem formulation}

Combining the vehicle lateral dynamics (9) and the vision system model (11) leads to a single dynamical system subject to the road curvature as a disturbance input and describing as follows:

$$
\left\{\begin{array}{l}
\dot{x}(t)=A x(t)+B u(t)+E d(t) \\
y(t)=C x(t)
\end{array}\right.
$$


with the state vector $x=\left[\begin{array}{llll}v_{y} & r & \psi_{L} & y_{L}\end{array}\right]^{T}$, the control input $u(t)=\delta_{f}$, the unknown input $d(t)=\rho$ and the matrices $A, B$, and $C$ defined by

$$
\begin{gathered}
A=\left[\begin{array}{cccc}
-\frac{c_{f}+c_{r}}{m v_{x}} & \frac{c_{r} l_{r}-c_{f} l_{f}}{m v_{x}}-v_{x} & 0 & 0 \\
\frac{c_{r} l_{r}-c_{f} l_{f}}{I_{z} v_{x}} & -\frac{c_{r} l_{r}^{2}+c_{f} l_{f}^{2}}{I_{z} v_{x}} & 0 & 0 \\
0 & 1 & 0 & 0 \\
1 & l_{s} & v_{x} & 0
\end{array}\right], \\
B=\left[\begin{array}{c}
\frac{c_{f}}{m} \\
\frac{c_{f} l_{f}}{I z} \\
0 \\
0
\end{array}\right], \quad E=\left[\begin{array}{c}
0 \\
0 \\
-v_{x} \\
-l_{s} v_{x}
\end{array}\right], \quad C=\left[\begin{array}{llll}
0 & 1 & 0 & 0 \\
0 & 0 & 1 & 0 \\
0 & 0 & 0 & 1
\end{array}\right]
\end{gathered}
$$

Note that the model (12) describing the vehicle lateral dynamics is subject to several variations and uncertainties. When road friction changes or when the nonlinear tire domain is reached, the tire forces $F_{y f}$ and $F_{y r}$ are no longer linearly proportional to slip angles due to the tire saturation property.

Taking into account this variation, the linear tire model (8) could correct the cornering stiffness by adding two uncertain terms $\Delta c_{f}$ and $\Delta c_{r}$ as:

$$
c_{f}=c_{f_{0}}+\Delta c_{f}, \quad c_{r}=c_{r_{0}}+\Delta c_{r}
$$

where the linear part, denoted by $c_{i 0}, \mathrm{i} \in\{f, r\}$, presents a known nominal value and the uncertainty term, denoted by $\Delta c_{i}, \mathrm{i} \in\{f, r\}$, is assumed to be unknown but bounded with a priori known bounds. Moreover, to deal with longitudinal velocity variations, a switched representation of the vehicle model is used and $v_{x}$ is assumed to be piecewise constant. Then, system (12) is transformed into a Switched Uncertain System given as follows:

$$
\left\{\begin{array}{l}
\dot{x}(t)=\left(A_{0, \sigma(t)}+\Delta A_{\sigma(t)}(\xi(t))\right) x(t)+ \\
\left(B_{0}+\Delta B(\xi(t))\right) u(t)+E_{\sigma(t)} d(t) \\
y(t)=C x(t)
\end{array}\right.
$$

where $x(t) \in \mathbb{R}^{n}, u(t) \in \mathbb{R}^{m}, y(t) \in \mathbb{R}^{p}, d(t) \in \mathbb{R}^{q}$ represent respectively the state, the control input, the output vector and the unknown input. $\xi(t)=\left[\begin{array}{ll}\Delta c_{f} & \Delta c_{r}\end{array}\right]^{T}$ is the vector of parameters uncertainty. $\sigma: \mathbb{R}^{+} \rightarrow$ $\mathcal{I}=\{1,2, \ldots, N\}$ is the switching signal. $A_{0, \sigma(t)}, B_{0}$, $E_{\sigma(t)}$ and $C$ are constant matrices of appropriate dimension. $\Delta A_{\sigma(t)}(\xi(t)) \in\left\{\Delta A_{1}(\xi(t)), \Delta A_{2}(\xi(t)), \ldots, \Delta A_{N}(\xi(t))\right\}$ and $\Delta B$ are bounded time-varying matrices. $N$ is the number of subsystems known a priori. For further developments, let define $\delta_{\sigma(t)}(t)=\Delta A_{\sigma(t)}(\xi(t)) x(t)+\Delta B(\xi(t)) u(t)$, the system (14) becomes

$$
\left\{\begin{array}{l}
\dot{x}(t)=A_{\sigma(t)} x(t)+B u(t)+E_{\sigma(t)} d(t)+\delta_{\sigma(t)}(t) \\
y(t)=C x(t)
\end{array}\right.
$$

\section{Performance Evaluation}

Vision systems use points interpolation to compute road curvature. However, this measure can be corrupted by different elements: occlusion, weather, noise on the image. We propose in the following a method to provide an observer based on board indicator for road curvature quality provided par the vision system. A block diagram of the evaluation procedure is illustrated in Figure 2. It includes:
1) A first switched interval observer which uses the measured variables, longitudinal velocity, yaw rate, steering angle, angular and offset displacements to estimate guaranteed bounds of the state vector considering the road curvature as unknown input;

2) A second interval observer based on the estimated bounds is built in order to reconstruct upper and lower bounds of the road curvature.

3) An evaluation block in which the road curvature measure is compared to the upper and lower estimated bounds.

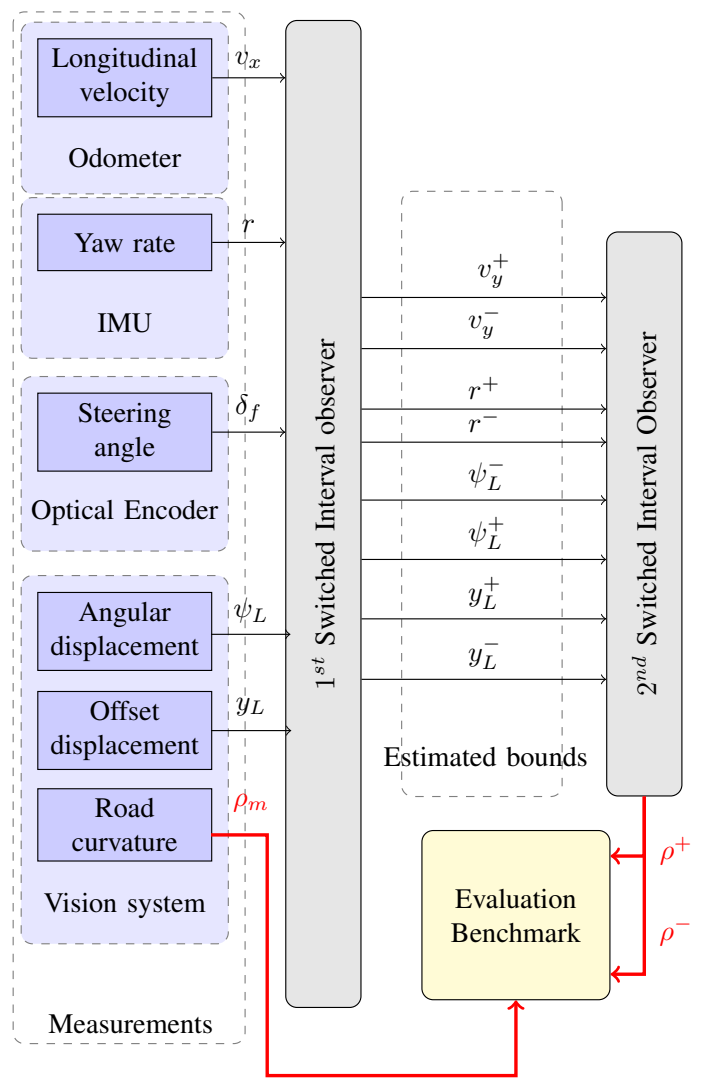

Fig. 2. Schematic overview of estimation methodology.

\section{A. SUIIO Design}

Consider the Switched Uncertain System described in (15). The aim is to design an Interval Observer to generate two trajectories $x^{-}(t)$ and $x^{+}(t)$ such that $x^{-}(t) \leq x(t) \leq x^{+}(t)$, $\forall t \geq t_{0}$ and $x^{-}\left(t_{0}\right) \leq x\left(t_{0}\right) \leq x^{+}\left(t_{0}\right)$ despite the presence of Unknown Input. To this end, the following assumptions are required.

Assumption 1. The state of the system $x(t)$ and the input vector $u(t)$ are all bounded.

Assumption 2. Assume that the input vector $u(t)$ is bounded as follows $u^{-}(t) \leq u(t) \leq u^{+}(t)$, such that $u^{-}(t)=u(t)-\zeta$ and $u^{+}(t)=u(t)+\zeta$, where $\zeta$ is a priori known bound.

Assumption 3. There exist known constants matrices $\Delta A_{\sigma(t)}^{+}$, $\Delta A_{\sigma(t)}^{-}, \Delta B^{+}, \Delta B^{-} \forall \sigma(t)$ such that:

$$
\Delta A_{\sigma(t)}^{-} \leq \Delta A_{\sigma(t)} \leq \Delta A_{\sigma(t)}^{+}
$$




$$
\Delta B^{-} \leq \Delta B \leq \Delta B^{+}
$$

Assumption 4. Assume that the rank condition $\operatorname{rank}\left(C E_{\sigma(t)}\right)=\operatorname{rank}\left(E_{\sigma(t)}\right), q \leq p$, holds $\forall \sigma(t)$.

Assumption 5. Assume that the following condition

$$
\operatorname{rank}\left(\left[\begin{array}{cc}
s \mathcal{I}_{n}-A_{\sigma(t)} & E_{\sigma(t)} \\
C & 0
\end{array}\right]\right)=n+q, \forall \sigma(t)
$$

holds for all complex number $s$ with $\mathcal{R} e(s) \geq 0$.

Assume that $x\left(t_{0}\right)^{-} \leq x\left(t_{0}\right) \leq x\left(t_{0}\right)^{+}$. Let us introduce observer gains matrices $N_{i} \in \mathbb{R}^{n \times n}, K_{i} \in \mathbb{R}^{n \times p}, H_{i} \in \mathbb{R}^{n \times p}$, $G_{i} \in \mathbb{R}^{n \times m}$ and $P_{i} \in \mathbb{R}^{n \times n} \forall i \in \mathcal{I}$, whose values will be specified later. Then, a Switched Unknown Input Interval Observer (SUIIO) structure for the lateral dynamics system (15) is given by:

$$
\left\{\begin{array}{l}
\dot{x}^{+}(t)=N_{\sigma(t)} x^{+}+K_{\sigma(t)} y+G_{\sigma(t)} u-H_{\sigma(t)} \dot{y}+ \\
\bar{P}_{\sigma(t)} \delta_{\sigma(t)}^{+}(t)-\underline{P}_{\sigma(t)} \delta_{\sigma(t)}^{-}(t) \\
\dot{x}^{-}(t)=N_{\sigma(t)} x^{-}+K_{\sigma(t)} y+G_{\sigma(t)} u-H_{\sigma(t)} \dot{y}+ \\
\bar{P}_{\sigma(t)} \delta_{\sigma(t)}^{-}(t)-\underline{P}_{\sigma(t)} \delta_{\sigma(t)}^{+}(t)
\end{array}\right.
$$

where $\delta_{\sigma(t)}^{+}(t)$ and $\delta_{\sigma(t)}^{-}(t)$ are defined using Definition 2, Assumptions 2 and 3 such that $\delta_{\sigma(t)}^{-}(t) \leq \delta_{\sigma(t)}(t) \leq \delta_{\sigma(t)}^{+}(t)$. The dynamics of the upper and lower estimation errors $e^{+}(t)$ and $e^{-}(t)$ is given by:

$$
\left\{\begin{array}{l}
\dot{e}^{+}(t)=N_{\sigma(t)} x^{+}(t)-\left(P_{\sigma(t)} A_{\sigma(t)}-K_{\sigma(t)} C\right) x(t)+ \\
\left(G_{\sigma(t)}-P_{\sigma(t)} B\right) u(t)-P_{\sigma(t)} E_{\sigma(t)} d(t)+\Delta_{\sigma(t)}^{+}(t) \\
\dot{e}^{-}(t)=\left(P_{\sigma(t)} A_{\sigma(t)}-K_{\sigma(t)} C\right) x(t)-N_{\sigma(t)} x^{-}(t)+ \\
\left(P_{\sigma(t)} B-G_{\sigma(t)}\right) u(t)+P_{\sigma(t)} E_{\sigma(t)} d(t)+\Delta_{\sigma(t)}^{-}(t)
\end{array}\right.
$$

where $P_{\sigma(t)}=H_{\sigma(t)} C+\mathcal{I}_{n}, \Delta_{\sigma(t)}^{+}(t)=\left(\bar{P}_{\sigma(t)} \delta_{\sigma(t)}^{+}(t)-\right.$ $\left.\underline{P}_{\sigma(t)} \delta_{\sigma(t)}^{-}(t)\right)-P_{\sigma(t)} \delta_{\sigma(t)}(t)$ and $\Delta_{\sigma(t)}^{-}(t)=P_{\sigma(t)} \delta_{\sigma(t)}(t)-$ $\left(\bar{P}_{\sigma(t)} \delta_{\sigma(t)}^{-}(t)-\underline{P}_{\sigma(t)} \delta_{\sigma(t)}^{+}(t)\right)$. Assume that the matrices $N_{\sigma(t)}, K_{\sigma(t)}, G_{\sigma(t)}$ and $H_{\sigma(t)}$ are solutions of:

$$
\begin{gathered}
N_{\sigma(t)}=P_{\sigma(t)} A_{0, \sigma(t)}-K_{\sigma(t)} C \\
G_{\sigma(t)}-P_{\sigma(t)} B=0 \\
P_{\sigma(t)}=H_{\sigma(t)} C+\mathcal{I}_{n} \\
H_{\sigma(t)}=-E_{\sigma(t)}\left(C E_{\sigma(t)}\right)^{\dagger}
\end{gathered}
$$

If the constrains (18) are fulfilled under Assumptions 4 and 5, which allows to cancel the unknown input $d(t)$, the dynamics of upper and lower estimation errors can be rewritten as:

$$
\left\{\begin{array}{l}
\dot{e}^{+}(t)=N_{\sigma(t)} e^{+}(t)+\Delta_{\sigma(t)}^{+}(t) \\
\dot{e}^{-}(t)=N_{\sigma(t)} e^{-}(t)+\Delta_{\sigma(t)}^{-}(t)
\end{array}\right.
$$

It is easy to verify that $\Delta_{\sigma(t)}^{+}(t) \geq 0$ and $\Delta_{\sigma(t)}^{-}(t) \geq 0, \forall i \in \mathcal{I}$ using Lemma 3. In addition, the initial state $x\left(t_{0}\right)$ verifies by construction $x^{-}\left(t_{0}\right) \leq x\left(t_{0}\right) \leq x^{+}\left(t_{0}\right)$. Thus, Definition 2 implies that $e^{+}(t) \geq 0$ and $e^{-(}(t) \geq 0$ if the gain $K_{\sigma(t)}$ is chosen such that $N_{\sigma(t)}$ is a Metzler matrix $\forall \sigma(t)$.

In the other hand, let define the interval error $e(t)=x^{+}(t)-$ $x^{-}(t)$, the dynamics of $e(t)$ is given by:

$$
\dot{e}(t)=N_{\sigma(t)} e(t)+\Delta_{\sigma(t)}(t)
$$

where $\Delta_{\sigma(t)}(t)=\Delta_{\sigma(t)}^{+}(t)-\Delta_{\sigma(t)}^{-}(t)$.

The robust state estimation problem is reduced to determine the observer gain $K_{\sigma(t)}$ such that the interval error $e(t)$ asymptotic convergence towards zero if $\Delta_{\sigma(t)}(t)=0, \forall \sigma(t)$ and to ensure an input-to-state stability (ISS) property of interval error with respect to the uncertainties in the case when $\Delta_{\sigma(t)}(t) \neq 0$.

Note that the conditions imposed to the gains matrix $K_{\sigma(t)}$ can be formalized in terms of Linear Matrix Inequalities using Piecewise Quadratic ISS-Lyapunov function of the form $V_{\sigma(t)}(e(t))=e^{T}(t) Q_{\sigma(t)}(t)$ described in Lemma 4. Then, it is required to find positive diagonal matrix $Q_{i}$, matrix $W_{i}$, $\beta>\alpha>0, \gamma>0$ for a given $\eta \geq 0, \varepsilon>0$, such that for all $i \in \mathcal{I}$,

$$
\begin{gathered}
\alpha \mathcal{I}_{n} \preceq Q_{i} \preceq \beta \mathcal{I}_{n} \\
{\left[\begin{array}{cc}
A_{0, i}{ }^{T} P^{T} Q_{i}^{T}-C^{T} W_{i}^{T}+ & \\
Q_{i} P A_{0, i}-W_{i} C+\varepsilon Q_{i} & Q_{i} \\
Q_{i} & -\gamma \mathcal{I}_{n}
\end{array}\right] \prec 0} \\
Q_{i} P A_{0, i}-W_{i} C+\eta Q_{i} \geq 0
\end{gathered}
$$

holds for the smallest value of $\gamma$, then the system (17) can estimate the lower and upper bounds of the state vector $x(t)$ with $K_{i}=Q_{i}^{-1} W_{i}$.

Furthermore the interval error (20) is Input-to-State Stable with respect to $\Delta_{\sigma(t)}(t)$ for any switching signal with Average Dwell Time satisfying condition (6), then if $\sup \left\|\Delta_{i}(t)\right\|_{\infty} \leq \Delta_{\max }$, (20) satisfies

$$
\lim _{t \rightarrow \infty} \sup \|e\| \leq \sqrt{\frac{\gamma}{\alpha \varepsilon}} \Delta_{\max }
$$

For brevity of presentation, all proofs are omitted.

\section{B. Unknown Input Interval reconstruction}

In this subsection, we will provide an unknown input interval reconstruction method based on previous estimated bounds of the state vector.

Under Assumptions 2-5, the Switched Unknown Disturbance Interval Observer (SUDIO) is built as follows:

$$
\left\{\begin{array}{l}
d^{+}(t)=\mathcal{K}_{\sigma(t)} y(t)-\mathcal{H}_{\sigma(t)} \dot{y}(t)+\mathcal{H}_{\sigma(t)} C B_{0, \sigma(t)} u(t) \\
+\varrho_{\sigma(t)}^{+}(t)-\left(\mathcal{K}_{\sigma(t)} C-\mathcal{H}_{\sigma(t)} C A_{0, \sigma(t)}\right) x^{-}(t) \\
d^{-}(t)=\mathcal{K}_{\sigma(t)} y(t)-\mathcal{H}_{\sigma(t)} \dot{y}(t)+\mathcal{H}_{\sigma(t)} C B_{0, \sigma(t)} u(t) \\
+\varrho_{\sigma(t)}^{-}(t)-\left(\mathcal{K}_{\sigma(t)} C-\mathcal{H}_{\sigma(t)} C A_{0, \sigma(t)}\right) x^{+}(t)
\end{array}\right.
$$

where $\mathcal{K}_{\sigma(t)}$ and $\mathcal{H}_{\sigma(t)}$ are the gains matrices to be designed. $\varrho_{\sigma(t)}^{+}(t)$ and $\varrho_{\sigma(t)}^{-}(t)$ are upper and lower bounds of $\varrho_{\sigma(t)}(t)=$ $\mathcal{H}_{\sigma(t)} C \delta_{\sigma(t)}(t)$ which can be constructed after determination of $\mathcal{H}_{\sigma(t)}$ using Lemma 2.

Let define the upper and lower estimation errors, $e_{d(t)}^{+}(t)=$ 
$d^{+}(t)-d(t)$ and $e_{d(t)}^{-}(t)=d(t)-d^{-}(t)$, from (25) and (15), it follows that

$$
\left\{\begin{array}{l}
e_{d(t)}^{+}(t)=-\mathcal{H}_{\sigma(t)} C\left(A_{0, \sigma(t)} x(t)+B_{0, \sigma(t)} u(t)+\right. \\
\left.E_{\sigma(t)} d(t)+\delta_{\sigma(t)}(t)\right)+\mathcal{K}_{\sigma(t)} C x(t)-\left(\mathcal{K}_{\sigma(t)} C\right. \\
\left.-\mathcal{H}_{\sigma(t)} C A_{0, \sigma(t)}\right) x^{-}(t)+\mathcal{H}_{\sigma(t)} C B_{0, \sigma(t)} u(t)+\varrho_{\sigma(t)}^{+}(t)-d(t) \\
e_{d(t)}^{-}(t)=\mathcal{H}_{\sigma(t)} C\left(A_{0, \sigma(t)} x(t)+B_{0, \sigma(t)} u(t)+\right. \\
\left.E_{\sigma(t)} d(t)+\delta_{\sigma(t)}(t)\right)-\mathcal{K}_{\sigma(t)} C x(t)+\left(\mathcal{K}_{\sigma(t)} C\right. \\
\left.-\mathcal{H}_{\sigma(t)} C A_{0, \sigma(t)}\right) x^{+}(t)-\mathcal{H}_{\sigma(t)} C B_{0, \sigma(t)} u(t)-\varrho_{\sigma(t)}^{-}(t)+d(t)
\end{array}\right.
$$

since $e^{+}(t)=x^{+}(t)-x(t)$ and $e^{-}(t)=x(t)-x^{-}(t)$, then,

$$
\left\{\begin{array}{l}
e_{d(t)}^{+}(t)=\left(\mathcal{K}_{\sigma(t)} C-\mathcal{H}_{\sigma(t)} C A_{0, \sigma(t)}\right) e^{-}(t) \\
-\left(\mathcal{I}_{q}+\mathcal{H}_{\sigma(t)} C E_{\sigma(t)}\right) d(t)+\Upsilon_{\sigma(t)}^{+}(t) \\
e_{d(t)}^{-}(t)=\left(\mathcal{K}_{\sigma(t)} C-\mathcal{H}_{\sigma(t)} C A_{0, \sigma(t)}\right) e^{+}(t) \\
+\left(\mathcal{I}_{q}+\mathcal{H}_{\sigma(t)} C E_{\sigma(t)}\right) d(t)+\Upsilon_{\sigma(t)}^{-}(t)
\end{array}\right.
$$

where $\Upsilon_{\sigma(t)}^{+}(t)=\varrho_{\sigma(t)}^{+}(t)-\varrho_{\sigma(t)}(t)$ and $\Upsilon_{\sigma(t)}^{-}(t)=\varrho_{\sigma(t)}(t)-$ $\varrho_{\sigma(t)}^{-}(t)$.

Note that $e^{+}(t) \geq 0, e^{-}(t) \geq 0, \Upsilon_{\sigma(t)}^{+}(t) \geq 0$ and $\Upsilon_{\sigma(t)}^{-}(t) \geq 0$ by construction. Furthermore, if the matrix $\left(\mathcal{K}_{\sigma(t)} C-\mathcal{H}_{\sigma(t)} C A_{0, \sigma(t)}\right)$ is nonnegative and the matrix $\mathcal{H}_{\sigma(t)}$ is chosen such that

$$
\mathcal{I}_{q}+\mathcal{H}_{\sigma(t)} C E_{\sigma(t)}=0
$$

then, the upper and lower errors (26) are positive $\forall \sigma(t)$, i.e. $e_{d(t)}^{+} \geq 0$ and $e_{d(t)}^{-} \geq 0$ which imply that

$$
d^{-}(t) \leq d(t) \leq d^{+}(t)
$$

If Assumption 4 holds, then a particular solution of (27) can be given as follows

$$
\mathcal{H}_{\sigma(t)}=-\mathcal{I}_{q}\left(C E_{\sigma(t)}\right)^{\dagger}
$$

Then, the switched unknown disturbance interval observer (25) estimate the upper and lower bounds of unknown input vector $d(t)$, i.e. (28) holds, if there exist matrices $\mathcal{K}_{i}, \forall i \in \mathcal{I}$ such that

$$
\mathcal{K}_{i} C-\mathcal{H}_{i} C A_{0, i} \geq 0, \quad \forall i \in \mathcal{I}
$$

\section{Evaluation System}

The goal is to use the two reliable computed bounds which are consistent with model structure, measurements and considered uncertainty in order to detect whether we can trust or not the measure given by the vehicle vision system. In fact, the proposed road curvature interval observer allows us to obtain curvature limits as they are reflected in the vehicle dynamics. In other words, the bounded error approach presented in this paper allow a characterization of the set of all admissible values of the road curvature. Therefore, one may test whether the interval includes the measured curvature or not

$$
\rho_{m} \in\left[\rho^{-}, \rho^{+}\right]
$$

If the constraint (31) holds, then the measurement provided by the vehicle onboard vision system is trustable. Otherwise, an abnormal situation appears which may correspond to a lane departure or a falsely measurement.

The design procedure of the evaluation benchmark can be summarized in the following design Algorithm 1.

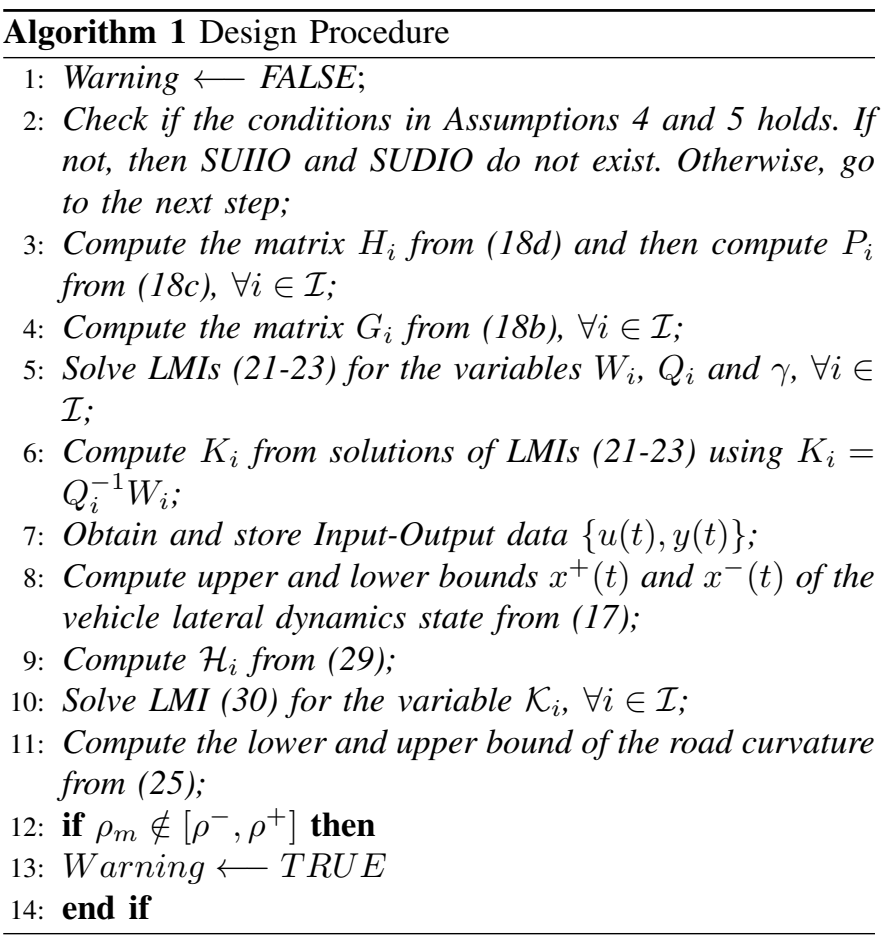

\section{Simulations Results}

In this section, the proposed evaluation methodology is applied to experimental data acquired using an instrumented vehicle. The yaw rate $r$ is measured using an inertial unit, the steering angle $\delta_{f}$ is measured by an absolute optical encoder while an odometer provides the vehicle longitudinal speed. A vision system provides road curvature, lateral offset and angular displacements measures. The steering angle, longitudinal velocity and vehicle trajectory profiles are shown in Figure 3. We assume that the cornering stiffness parameters are affected by $10 \%$ uncertainty in their nominal value. As mentioned above, the switching law $\sigma(t)$ depends on the varying longitudinal velocity $v_{x}$ which is accessible in real time, such that:

$$
\sigma(t)=\left\{\begin{array}{lll}
1 & \text { if } & v_{x} \in\left[V_{x}^{0}, V_{x}^{1}[\right. \\
2 & \text { if } v_{x} \in\left[V_{x}^{1}, V_{x}^{2}[\right. \\
3 & \text { if } v_{x} \in\left[V_{x}^{2}, V_{x}^{3}\right]
\end{array}\right.
$$

with $v_{x}^{k}=\frac{V_{x}^{k}-V_{x}^{k-1}}{2}$ for $k=1,2,3$.

The numerical simulation was carried out by using Matlab optimization tools (Yalmip or Sedumi). Due to space limitation, the gains matrices are omitted and the results of interval estimation of the lateral velocity, yaw rate, offset and angular displacements are depicted in Figure 4. The curvature measure and the corresponding upper and lower bounds are shown in Figure 5. It should be noted that in order to test the performance of the Algorithm 1, inaccurate measurements of the road curvature are generated during data acquisition from time instant 83 to 90.5 (light blue zone) by ironing hand in front of the camera. As shown in Figure 5, the test criteria (31) is satisfied outside the faulty zone, demonstrating the reliability of the measurement. Whereas, zooming on the light blue area, 
shows that the curvature is sometimes outside the estimated interval proving that the measurement is not trustable.
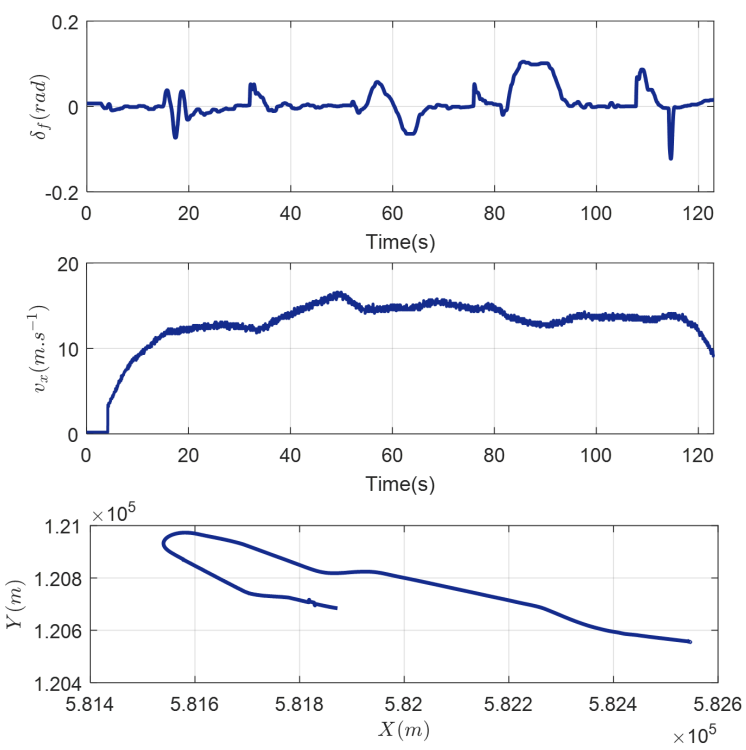

Fig. 3. Steering angle, Longitudinal velocity and Vehicle trajectory.
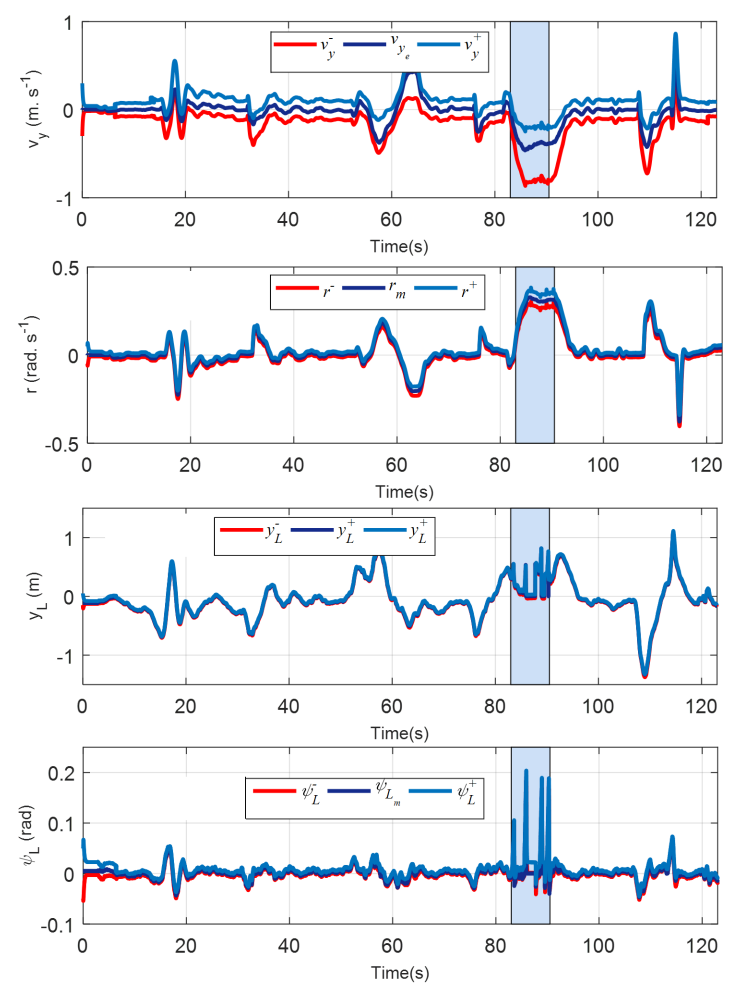

Fig. 4. Interval estimation of lateral velocity, yaw rate, offset and angular displacements.

\section{CONCLUSION}

An evaluation methodology of vehicle onboard vision system has been presented in this paper. The fundamental evaluation is made by estimating the guaranteed lower and upper
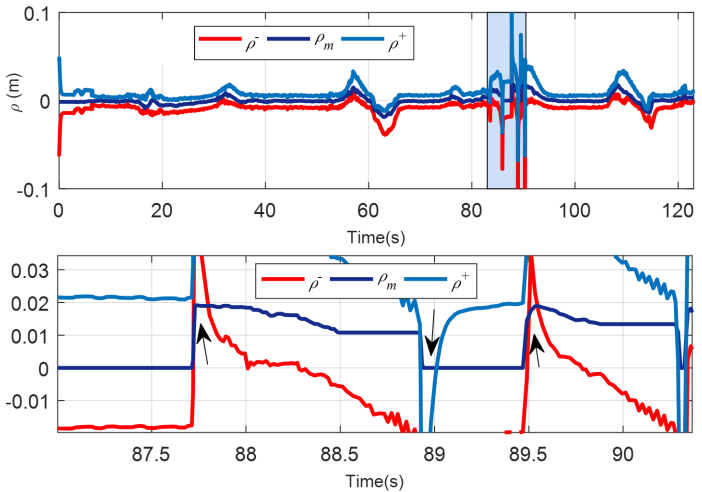

Fig. 5. Interval estimation of road curvature with a zoomed view of the light blue zone.

bounds for vehicle-state at any instant. The method takes into account the variations which might occur during the vehicle maneuver. Performance of the proposed Algorithm is evaluated using vehicle real data, simulation results demonstrate that the proposed evaluation scheme succeeds to appropriately estimate the upper and lower bounds of vehicle lateral dynamics and road curvature.

\section{REFERENCES}

[1] Rajamani, R. (2011). Vehicle dynamics and control. Springer Science \& Business Media.

[2] Dugoff, H., Fancher, P. S., \& Segel, L. (1970). An analysis of tire traction properties and their influence on vehicle dynamic performance (No. 700377). SAE Technical Paper.

[3] Pacejka, H. B., \& Bakker, E. (1992). The magic formula tyre model. Vehicle system dynamics, 21(S1), 1-18.

[4] Gucik-Derigny, D., Rassi, T., \& Zolghadri, A. (2014). Interval state and unknown inputs estimation for linear time-invariant systems. IFAC Proceedings Volumes, 47(3), 7375-7381.

[5] Ifqir, S., Ait-Oufroukh, N., Ichalal, D., \& Mammar, S. (2017, July). Synchronous interval observer design for switched LPV systems using multiple quadratic ISS-Lyapunov functions. IEEE. In Control and Automation (MED), 2017 25th Mediterranean Conference on (pp. 388393).

[6] Efimov, D., Fridman, L., Raissi, T., Zolghadri, A., \& Seydou, R. (2012). Interval estimation for LPV systems applying high order sliding mode techniques. Automatica, 48(9), 2365-2371.

[7] Minc, H. (1988). Nonnegative matrices (p. 152). J. Wiley.

[8] Rami, M. A., Cheng, C. H., \& De Prada, C. (2008, December). Tight robust interval observers: an LP approach. 47th IEEE Conference on Decision and Control. CDC 2008. (pp. 2967-2972).

[9] Blanchini, F., Colaneri, P., \& Valcher, M. E. (2015). Switched linear positive systems. Found. Trends Syst. Control, 2(2), 101-273.

[10] Mammar, S., Glaser, S., \& Netto, M. (2006). Time to line crossing for lane departure avoidance: A theoretical study and an experimental setting. IEEE Transactions on Intelligent Transportation Systems, 7(2), 226-241.

[11] Ifqir, S., Ait-Oufroukh, N., Ichalal, D., \& Mammar, S. Switched unknown inputs interval observer design for vehicle lateral dynamics estimation with wind gusts rejection. In : Proc. 14th International Workshop on Advanced Control and Diagnosis, (2017).

[12] Ifqir, S., Ait-Oufroukh, N., Ichalal, D., \& Mammar, S. Robust interval observer for switched systems with unknown inputs : application to vehicle dynamics estimation. To be published in European Journal of Control [Preprint], 2018.

[13] Efimov, D., Rassi, T., Chebotarev, S., \& Zolghadri, A. (2013). Interval state observer for nonlinear time varying systems. Automatica, 49(1), 200-205. 